FECHA DE

PUBLICACIÓN:

$10-05-2021$

FECHA DE

ACEPTACIÓN:

20-04-2021

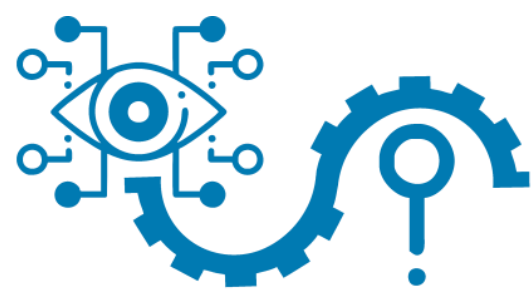

GnasisWisdam
REVISTA DE INVESTIGACIÓN E INNOVACIÓN CIENTÍFICA Y TECNOLÓGICA

Artículo original

Volumen 1, Número 2, Mayo - Agosto 2021

\title{
SISTEMA DE SEGUIMIENTO DE EGRESADOS DE LA FACULTAD DE INGENIERÍA ELECTRÓNICA - SISTEMAS DE LA UNIVERSIDAD NACIONAL DE HUANCAVELICA, SEDE PAMPAS
}

\section{GRADUATE MONITORING SYSTEM OF THE FACULTY OF ELECTRONIC - SYSTEMS ENGINEERING - OF THE NATIONAL UNIVERSITY OF HUANCAVELICA PAMPAS}

\section{Autores}

Freddy Toribio, Huayta Meza

Universidad Nacional de Huancavelica

Luis Enrique, Pacheco Moscoso ORCID

Universidad Nacional de Huancavelica

Noemi, Aquino Sinche ORCID

Universidad Nacional Autónoma de Tayacaja Daniel Hernández Morillo

\section{Resumen}

La implementación de un "Sistema de Seguimiento de Egresados" en la Facultad de Ingeniería Electrónica - Sistemas de la Universidad Nacional de Huancavelica - Sede Pampas, tuvo como objetivo desarrollar e implementar un sistema automatizado de seguimiento de egresados a fin de que se pueda conocer su ubicación e inserción en el mercado laboral, su formación continua (Ley Universitaria $\mathrm{N}^{\circ} 30220$ Art. 46), la vinculación con la empresas y el aporte que los graduados puedan ofrecer a la facultad en la mejora de su oferta en la carrera profesional. La investigación fue del tipo descriptivo y la muestra es no probabilística por que, a través de los correos electrónicos de los egresados se envió los cuestionarios para estos fuesen rellenados voluntariamente y enviados al correo institucional. Para el procesamiento de datos se utilizó el programa Statistical Package for the Social Sciences (SPSS), Para el desarrollo del Sistema de Seguimiento de Egresados se empleó la metodología Extreme Programming (XP) que es la metodología más destacada de los procesos ágiles de desarrollo de software, para la programación Appeon Power Builder 2017, y para el modelamiento del sistema el Unified Modeling Language (UML). El Sistema, permite registrar y mantener actualizado sus datos personales y académicos, modalidad de trabajo (independiente/dependiente), datos de las empresas empleadoras, nivel de satisfacción de los empleadores para con los egresados. Así mismo, permitirá analizar la información obtenida y tomar decisiones en cuanto a la oferta académica de la Facultad de Ingeniería Electrónica - Sistemas. Para la evaluación del sistema se utilizó el System Usability Scale (SUS) cuyo resultado promedio fue de 71.6, y se encuentra dentro del rango de "Bueno", que significa que el usuario encuentra satisfacción en el empleo del sistema, pudiéndose realizar algunas mejoras. 


\title{
GnasisWisdam
}

Palabras claves: Sistema, seguimiento de egresados, mercado laboral, nivel de satisfacción, toma de decisiones.

\begin{abstract}
The implementation of a "Graduate Monitoring System" in the Faculty of Electronic - Systems Engineering of the National University of Huancavelica - Pampas Headquarters, was aimed at developing and implementing an automated system for monitoring graduates so that their location and insertion in the labor market, its continuous training (University Law No. 30220 Art. 46), the link with companies and the contribution that graduates can offer to the faculty in improving their offer in the professional career. The investigation was of the descriptive type and the sample is not probabilistic because, through the emails of the graduates, the questionnaires for these were voluntarily filled out and sent to the institutional mail. For the data processing the Statistical Package for the Social Sciences (SPSS) program was used, for the development of the Graduate Tracking System the Extreme Programming (XP) methodology was used, which is the most outstanding methodology of the agile processes of development of software, for the Appeon Power Builder 2017 programming, and for the system modeling the Unified Modeling Language (UML). The System allows you to register and keep your personal and academic data updated, work modality (independent / dependent), data of the employer companies, level of satisfaction of the employers towards the graduates. Likewise, it will allow analyzing the information obtained and making decisions regarding the academic offer of the Faculty of Electronic - Systems Engineering. For the evaluation of the system, the System Usability Scale (SUS) was used, the average result of which was 71.6, and is within the "Good" range, which means that the user finds satisfaction in the use of the system, being able to make some improvements.
\end{abstract}

Keywords: System, follow-up of graduates, labor market, level of satisfaction, decision making.

\section{Introducción}

En la perspectiva de lograr el objetivo de mejora continua de las carreras profesionales, hoy en día resulta importante mantener comunicación con sus egresados para conocer el nivel de inserción en el mercado laboral, saber si su rendimiento laboral y académico es adecuado de acuerdo al perfil de egreso de la carrera profesional.

Asimismo, la Facultad de Ingeniería Electrónica - Sistemas está en pleno proceso de acreditación, el cual tiene como uno de sus objetivos realizar el seguimiento a sus egresados para saber el tiempo que demoran en insertarse en el mercado laboral y conocer 
Volumen 1, Número 2, Mayo-Agosto 2021 Fecha de publicación: 10-05-2021 - Fecha de aceptación:20-04-2021

\section{GnasisWisdam}

si su rendimiento laboral y académico es el adecuado.

En tal sentido, se evidencia la necesidad de implementar un sistema de seguimiento de egresados que coadyuve a la articulación entre la oferta educativa y la demanda laboral y posibilite el mejoramiento de la calidad de la Facultad a partir de los resultados de la valoración del desempeño laboral desde la óptica de los empleadores y de los egresados de la Facultad de Ingeniería Electrónica - Sistemas.

Por tanto, es a través de los sistemas de seguimiento de egresados que se busca tener información real acerca de las actividades en las que se desempeñan nuestros egresados de la Facultad de Ingeniería Electrónica Sistemas, el grado de empleabilidad que alcanzan, los salarios que perciben, cuantos laboran de manera independiente en la especialidad, el nivel de preparación que tienen para el desempeño de las tareas profesionales, entre otras.

Asimismo, se pueda conocer la opinión de los empleadores en cuanto al desempeño profesional, las competencias y capacidades que están adecuadamente desarrolladas, así como aquellas que requieren reforzamiento y en qué medida lo requieren.

Para el desarrollo del proyecto, se, utilizó la metodología Extreme Programming. (XP). (juego de la planificación, entregas pequeñas, uso de metáforas, pruebas, sencillez, programación en parejas, refactorización, cliente en sitio, entre otras), así mismo, se hizo el levantamiento de requerimientos (carpeta académica de egresados), tareas de ingeniería y pruebas de usabilidad de software, que dieron pie a un sistema automatizado que funciona bajo la plataforma Windows, compilado en el lenguaje Appeon Poer Builder 2017. Y finalmente, se realizó las pruebas necesarias para verificar y validar el funcionamiento correcto del sistema.

\section{Material y Métodos}

La investigación realizada es de tipo descriptivo, ya que busca conocer la estructura fundamental de todos los procesos del sistema de seguimiento a egresados, describiendo la situación actual de estos procesos, de manera que se puedan especificar sus características y propiedades necesarias para la automatización de los mismos.

\section{Población y Muestra}

En este proyecto la población estuvo representada por los estudiantes egresados o graduados a partir del año 2010 de la Facultad de Ingeniería Electrónica Sistemas de la Universidad Nacional de Huancavelica - Sede Pampas. Asimismo, por las empresas empleadoras en las cuales laboran los egresados.

La muestra es no probabilística ya que solo se consideró a los egresados que rellenaron y enviaron el cuestionario al correo institucional de la Facultad. 


\section{REVISTA DE INVESTIGACIÓN E INNOVACIÓN}

CIENTÍFICA Y TECNOLÓGICA

Volumen 1, Número 2, Mayo-Agosto 2021

Fecha de publicación: 10-05-2021 - Fecha de aceptación:20-04-2021

\section{GnasisWisdam}

Técnicas de recolección de datos

Para el desarrollo del proyecto, primero se coordinó con el Área Académica de las Escuelas Profesionales de Ingeniería Electrónica e Ingeniería de Sistemas, para que proporcionen los files de los estudiantes egresados a partir del año 2010 y así poder obtener sus números de celulares y/o correos electrónicos para poder comunicarnos y enviar el cuestionario a través de sus correos electrónicos para que estos puedan ser rellenados voluntariamente y reenviados al correo institucional de la Facultad.

De los cuestionarios enviados por los egresados se obtuvieron los datos de los empleadores, a quienes se les llamó y se solicitó sus correos electrónicos para remitir la encuesta y ellos pudieran reenviar el cuestionario rellenado al correo institucional, esto sirvió para conocer el grado de satisfacción para con nuestros egresados.

\section{Diseño Operativo}

Para el desarrollo del proyecto, se siguió un diseño operativo derivado del ciclo de vida de la metodología de desarrollo para sistemas de información llamada Extreme Programming o XP (Kent Beck, 1996), el cual consta de 6 fases iterativas: Exploración, Planificación de la Entrega, Iteraciones, Producción, Mantenimiento $\mathrm{y}$ Muerte del Proyecto.

Partiendo de lo anterior, se agruparon las dos primeras fases metodológicas de XP en una sola, y así darle paso, a las siguientes cinco (05) fases iterativas (y en parte simultaneas) del proyecto; todas necesarios para cumplir con el objetivo planteado, poniendo en marcha los valores, principios y prácticas de $\mathrm{XP}$, tal como se muestra en el siguiente cuadro operativo.

\begin{tabular}{|c|c|c|c|}
\hline METODOLOGÍA & OBJETIVOS ESPECÍFICOS & ACTIVIDADES & FASES \\
\hline $\begin{array}{c}\text { Extreme } \\
\text { Programming } \\
\text { (XP) }\end{array}$ & $\begin{array}{l}\text { Identificar la situación problemática del Sistema } \\
\text { de seguimiento a egresados. } \\
\text { Automatizar el registro de información de los } \\
\text { egresados y empleadores. }\end{array}$ & $\begin{array}{l}\text { 1. Hacer una observación general de las } \\
\text { actividades llevadas a cabo. } \\
\text { 2. Aplicar el cuestionario a los egresados y } \\
\text { empleadores. } \\
\text { 3. Definir Objetivos: }\end{array}$ & $\begin{array}{c}\text { 1era Fase: } \\
\text { Exploración y } \\
\text { Planificación } \\
\text { del Proyecto. }\end{array}$ \\
\hline $\begin{array}{c}\text { Extreme } \\
\text { Programming } \\
\text { (XP) }\end{array}$ & $\begin{array}{l}\text { Elaborar de acuerdo a su importancia la } \\
\text { construcción del sistema de seguimiento a } \\
\text { egresados } \\
\text { Diseñar un sistema de seguimiento a egresados } \\
\text { que incluye a los empleadores, opiniones y } \\
\text { sentimiento. }\end{array}$ & $\begin{array}{l}\text { 6. Reuniones con los usuarios en intervalos } \\
\text { pequeños para establecer prioridades } \\
\text { 7. Delimitar el alcance del proyecto, junto con los } \\
\text { objetivos para lograr el desarrollo del sistema } \\
\text { de seguimiento a egresados }\end{array}$ & $\begin{array}{l}\text { 2da fase: } \\
\text { Producción y } \\
\text { Diseño del } \\
\text { proyecto }\end{array}$ \\
\hline $\begin{array}{c}\text { Extreme } \\
\text { Programming } \\
\text { (XP) }\end{array}$ & $\begin{array}{l}\text { Construir el sistema automatizado, de manera } \\
\text { ordenada través de códigos que sean entendibles y } \\
\text { fáciles de modificar en un momento dado evitando } \\
\text { incrementar costos y gastos innecesarios. } \\
\text { Realizar pruebas continuamente mediante el } \\
\text { diseño del sistema para la verificación de su } \\
\text { correcto desarrollo. }\end{array}$ & $\begin{array}{l}\text { 8. Diseños de Software Entregas pequeñas del } \\
\text { sistema } \\
\text { 9. Codificar e ir corrigiendo y haciendo pruebas } \\
\text { del sistema junto con el cliente. } \\
\text { 10. Por último, realizar un manual de usuarios y } \\
\text { ejecutar el programa para explicarle de } \\
\text { manera detallada al cliente sus funciones }\end{array}$ & $\begin{array}{c}\text { 3era: } \\
\text { Codificación y } \\
\text { Mantenimient } \\
\text { o del proyecto }\end{array}$ \\
\hline
\end{tabular}


Volumen 1, Número 2, Mayo-Agosto 2021 Fecha de publicación: 10-05-2021 - Fecha de aceptación:20-04-2021

\section{GnosisWisdam}

\section{Resultados}

El proyecto consiste en desarrollar un sistema de seguimiento de egresados, que recopile información sobre los egresados de la Facultad de Ingeniería de Electrónica Sistema.

Partiendo del diseño operativo, los resultados, productos de la aplicación de la metodología de desarrollo XP, se muestran por medio de tres (03) etapas o fases, Exploración y Planificación del proyecto, Producción y Diseño del proyecto, y Codificación y Mantenimiento del proyecto.

Implementación del Sistema de Seguimiento de Egresados de la Facultad de Ingeniería Electrónica - Sistemas

Figura 1. Diagrama de flujo de seguimiento de egresado

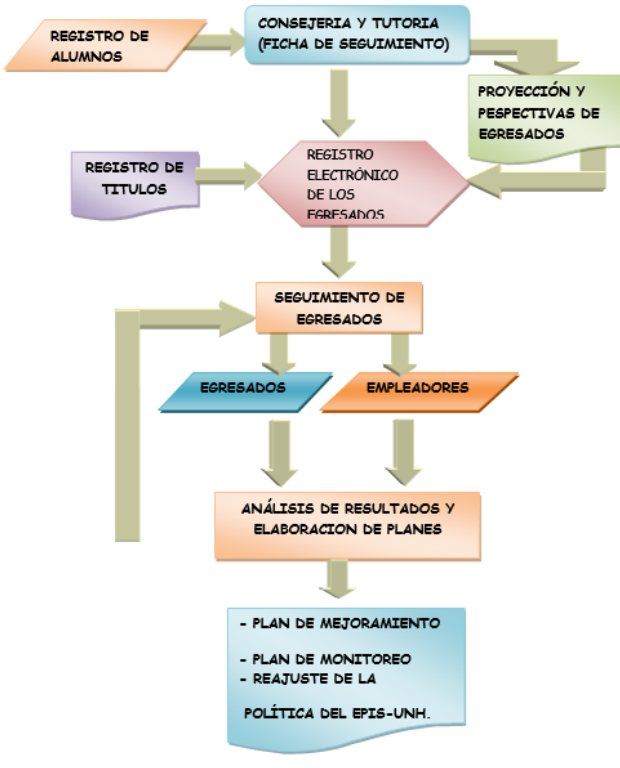

El diagrama de flujo facilitó la comprensión de las actividades realizadas en el departamento, lo que permitió la selección de los criterios o factores para el desarrollo del sistema. Debido a que los procesos indicados no sufrieron ningún tipo de modificación, éste se mantuvo con validez para el desarrollo del sistema.

Tablas de historias de usuario.

\section{Requerimientos para la primera iteración}

Las tablas 1 y 2 hacen referencia a la información almacenada y vinculada a los egresados y a los empleadores.

Tabla 1. Historia de Usuario $\mathrm{N}^{\circ} 1$.

\begin{tabular}{|c|c|c|c|}
\hline \multicolumn{4}{|c|}{ HISTORIA DE USUARIO $\mathrm{N}^{\circ} 1$} \\
\hline $\begin{array}{l}\text { Prioridad } \\
\text { del negocio: }\end{array}$ & $\begin{array}{l}\mathrm{Al} \\
\mathrm{ta}\end{array}$ & $\begin{array}{l}\text { Nomb } \\
\text { re: }\end{array}$ & $\begin{array}{l}\text { Registrar datos del } \\
\text { egresado }\end{array}$ \\
\hline $\begin{array}{l}\text { Puntos } \\
\text { estimados }\end{array}$ & 9 & $\begin{array}{l}\text { Usuar } \\
\text { io: }\end{array}$ & $\begin{array}{ll}\text { Responsable } & \text { de } \\
\text { Seguimiento } & \text { de } \\
\text { Egresado } & \end{array}$ \\
\hline $\begin{array}{l}\text { Iteración } \\
\text { Asignada }\end{array}$ & 1 & $\begin{array}{l}\text { Progr } \\
\text { amad } \\
\text { or } \\
\text { respo } \\
\text { nsable }\end{array}$ & Investigadores \\
\hline \multicolumn{4}{|c|}{$\begin{array}{l}\text { Se ingresa los datos del egresado como: } \mathrm{N}^{\circ} \text { de } \mathrm{DNI} \text {, } \\
\text { apellidos, nombres, e-mail, celular, teléfono fijo, } \\
\text { promoción, turno; si se ingresa dos veces el mismo } \\
\mathrm{N}^{\circ} \text { DNI debe arrojarme error. }\end{array}$} \\
\hline OBSERVA & ION & & \\
\hline
\end{tabular}

Tabla 2. Historia de usuario Nro. 2

\begin{tabular}{|c|c|c|c|}
\hline \multicolumn{4}{|c|}{ PRUEBA DE ACEPTACIÓN N ${ }^{\circ} 1$} \\
\hline $\begin{array}{ll}\text { Número de } \\
\text { caso de } \\
\text { prueba }\end{array}$ & 1 & $\begin{array}{l}\text { Nomb } \\
\text { re del } \\
\text { caso } \\
\text { de } \\
\text { prueb } \\
\text { a }\end{array}$ & $\begin{array}{l}\text { Verificar egresado } \\
\text { registrado }\end{array}$ \\
\hline $\begin{array}{ll}N^{\circ} & \text { de } \\
\text { historia de } \\
\text { usuario }\end{array}$ & 1 & $\begin{array}{l}\text { Usuar } \\
\text { io: }\end{array}$ & $\begin{array}{ll}\text { Responsable } & \text { de } \\
\text { Seguimiento } & \text { de } \\
\text { Egresado } & \end{array}$ \\
\hline
\end{tabular}




\section{GnasisWisdam}

DESCRIPCIÓN:
Se ingresa el número de DNI, si el número ya
existe mostrar error dando un mensaje "Egresado
ya registrado".
OBSERVACIONES:

Tareas de Ingeniería de la primera iteración

La primera iteración consta de dos historias de usuario, las cuales se satisfacen según las dos tareas de ingeniería.

Tabla 3. Tarea de Ingeniería $\mathrm{N}^{\circ} .1$

\begin{tabular}{|l|l|}
\hline \multicolumn{2}{|c|}{ Tarea de Ingeniería } \\
\hline $\mathbf{N}^{\circ} \mathbf{1}$ & $\begin{array}{l}\text { Historia de Usuario } \mathbf{N}^{\circ} \\
\text { 1: Registrar datos del } \\
\text { egresado }\end{array}$ \\
\hline $\begin{array}{l}\text { Nombre de la } \\
\text { Tarea: }\end{array}$ & Puntos Estimados: 1 \\
\hline $\begin{array}{l}\text { Tipo } \\
\text { Tarea: }\end{array}$ & de \\
\hline $\begin{array}{l}\text { Tiempo } \\
\text { Trabajo: } \\
\text { semana }\end{array}$ & $\begin{array}{l}\text { Programador } \\
\text { responsable: }\end{array}$ \\
\hline $\begin{array}{l}\text { Investigadores } \\
\text { Descripción: }\end{array}$ \\
$\begin{array}{l}\text { Se ingresa los datos del egresado como: } \\
\mathrm{N}^{\circ} \text { de DNI, apellidos, nombres, e-mail, } \\
\text { celular, teléfono fijo, promoción, turno; si } \\
\text { se ingresa dos veces el mismo } \mathrm{N}^{\circ} \text { DNI } \\
\text { debe arrojarme error }\end{array}$ \\
\hline
\end{tabular}

Tabla 4. Tarea de Ingeniería $\mathrm{N}^{\circ} .2$

\begin{tabular}{|l|l|}
\hline \multicolumn{2}{|c|}{ Tarea de Ingeniería } \\
\hline $\mathrm{N}^{\circ} 2$ & $\begin{array}{l}\text { Historia de Usuario } \mathrm{N}^{\circ} \\
\text { 2: Registrar datos del } \\
\text { empleador }\end{array}$ \\
\hline $\begin{array}{l}\text { Nombre de la } \\
\text { Tarea: }\end{array}$ & Puntos Estimados: 1 \\
\hline
\end{tabular}

\begin{tabular}{|l|l|}
\hline $\begin{array}{l}\text { Tipo } \\
\text { Tarea }\end{array}$ & de \\
$\begin{array}{l}\text { Tiempo de } \\
\text { Trabajo: } \\
\text { semanas }\end{array}$ & $\begin{array}{l}\text { Programador } \\
\text { responsable: } \\
\text { Investigadores }\end{array}$ \\
\hline $\begin{array}{l}\text { Descripción: Se ingresa los datos del } \\
\text { empleador como: No de RUC, Nombre de } \\
\text { la empresa, Dirección, Teléfono, Celular, } \\
\text { Tipo de empresa, e-mail, web, nombre } \\
\text { del gerente, DNI del gerente. Si ingresa } \\
\text { un número de RUC existente, debe } \\
\text { mostrar un mensaje de error }\end{array}$ \\
\hline
\end{tabular}

Tareas de Ingeniería de la segunda iteración

Fueron elaboradas dos tareas para la iteración número dos, las cuales fueron desarrolladas paralelamente a la anterior.

Tabla 5. Tarea de Ingeniería $\mathrm{N}^{\circ} \cdot 3$

\begin{tabular}{|c|c|}
\hline \multicolumn{2}{|c|}{ Tarea de Ingeniería } \\
\hline $\mathbf{N}^{\circ} \mathbf{3}$ & $\begin{array}{lr}\text { Historia de Usuario } \mathbf{N}^{\circ} \\
\text { 3: } & \text { Registrar } \\
\text { información } & \text { de } \\
\text { egresados } & \end{array}$ \\
\hline $\begin{array}{l}\text { Nombre de la } \\
\text { Tarea: }\end{array}$ & Puntos E \\
\hline $\begin{array}{l}\text { Tipo } \\
\text { Tarea }\end{array}$ & \\
\hline $\begin{array}{lr}\text { Tiempo } & \text { de } \\
\text { Trabajo: } & 1 \\
\text { semana } & \end{array}$ & $\begin{array}{l}\text { Programador } \\
\text { responsable: } \\
\text { Investigadores }\end{array}$ \\
\hline \multicolumn{2}{|c|}{$\begin{array}{l}\text { Descripción: Se ingresa la información de } \\
\text { egresado; solo si el egresado ha side } \\
\text { registrado en el sistema, o sea; se ingresa su } \\
\mathrm{N}^{\circ} \mathrm{DNI} \text { en caso de no estar registrado deb } \\
\text { enviar un mensaje de error, luego la } \\
\text { siguiente información }\end{array}$} \\
\hline
\end{tabular}

Tareas de Ingeniería de la tercera iteración 


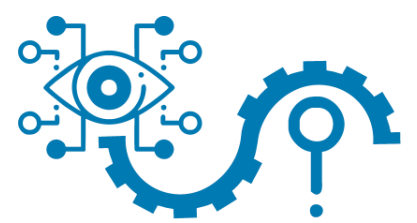

\section{GnasisWisdam}

Fueron elaboradas dos tareas para el desarrollo y cumplimiento de los requerimientos de la tercera iteración.

Figura 2. Diagrama a nivel de contexto del Sistema de Seguimiento a Egresados

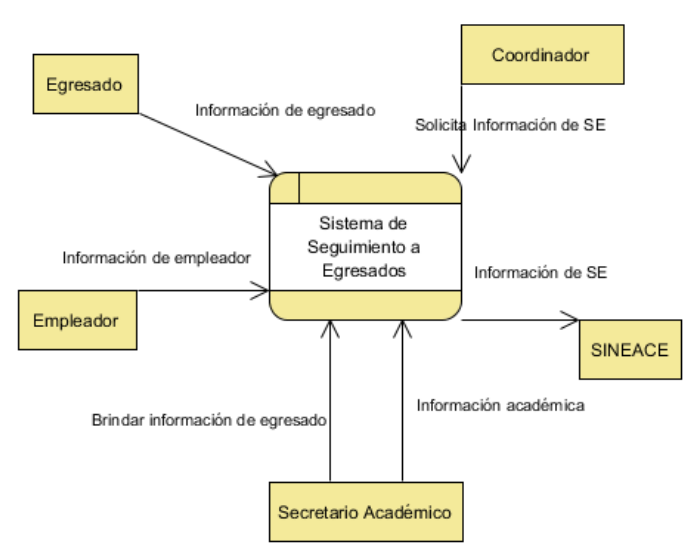

Figura 3. Diagrama a nivel de hijos

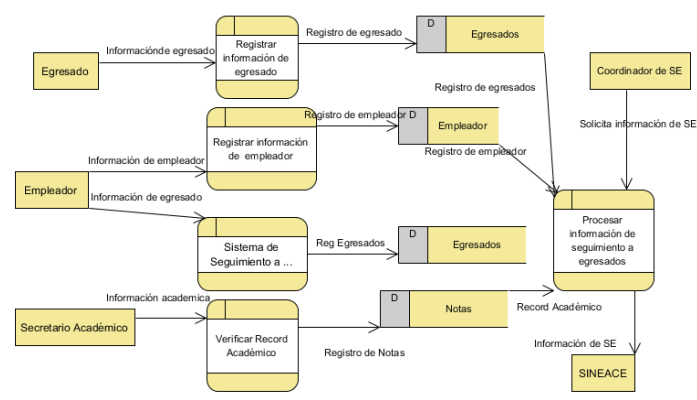

Modelo de base de datos

Figura 4. Esquema Relacional de la base de datos

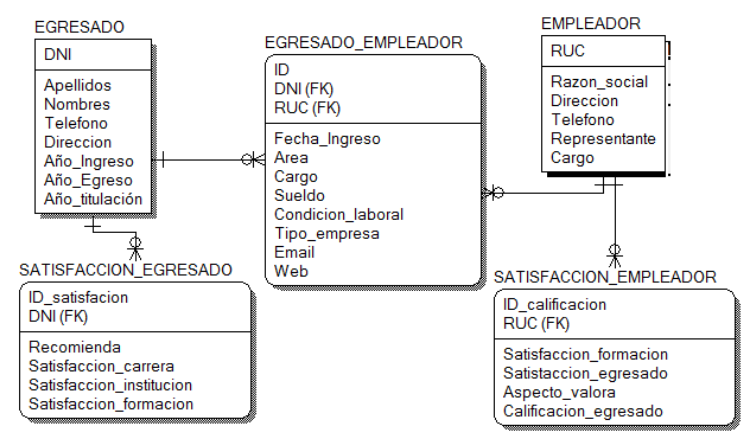

Diseño de la base de datos

\section{REVISTA DE INVESTIGACIÓN E INNOVACIÓN}

CIENTÍFICA Y TECNOLÓGICA

Volumen 1, Número 2, Mayo-Agosto 2021 Fecha de publicación: 10-05-2021 - Fecha de aceptación:20-04-2021
Figura 5. Modelo Entidad - Relación de la base de datos

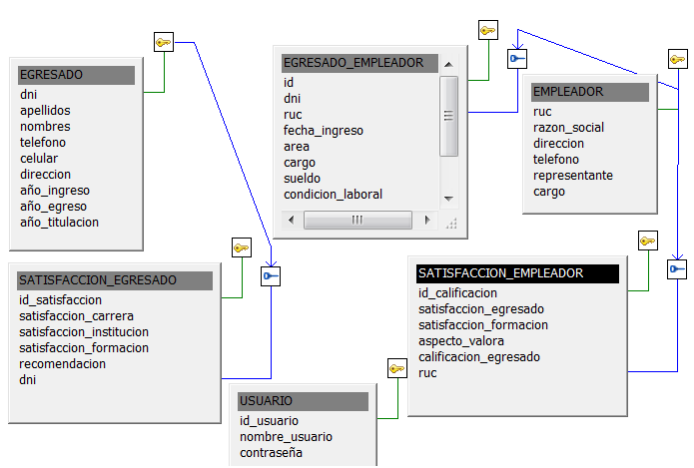

Diagrama de procesos de Seguimiento a

Egresados

Figura 6. Diagrama de procesos - Sistema de seguimiento a egresados

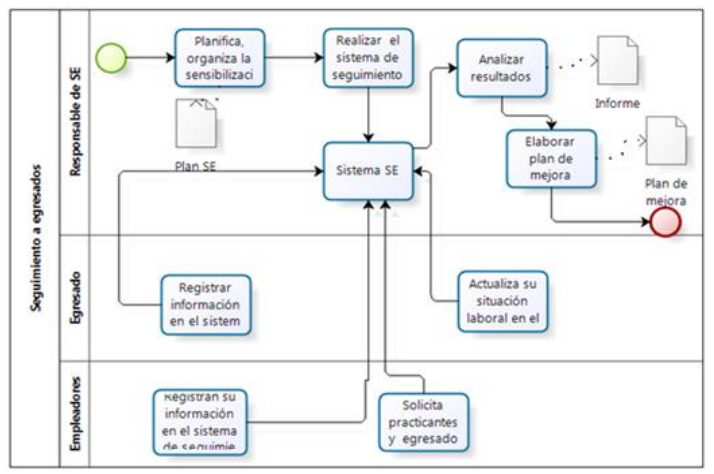

\section{Modelo de Casos de Uso del Sistema}

Para obtener el resultado deseado por el usuario, el sistema lleva a cabo una serie de acciones, las cuales pueden ser o no ser dependientes unas de otras. Para conocer dichas acciones se desarrolló un modelo general de 8 casos de uso (CU) del sistema, y su posterior desglose en casos de usos más simples facilitó la comprensión de los procesos que se ejecutan con cada solicitud o clic efectuado por el usuario. A continuación, se muestra el diagrama general de caso de uso del sistema. 


\section{REVISTA DE INVESTIGACIÓN E INNOVACIÓN \\ CIENTÍFICA Y TECNOLÓGICA}

Volumen 1, Número 2, Mayo-Agosto 2021

Fecha de publicación: 10-05-2021 - Fecha de aceptación:20-04-2021

\section{GnasisWisdam}

Figura 7. Diagrama de CU general del

sistema de seguimiento a egresados

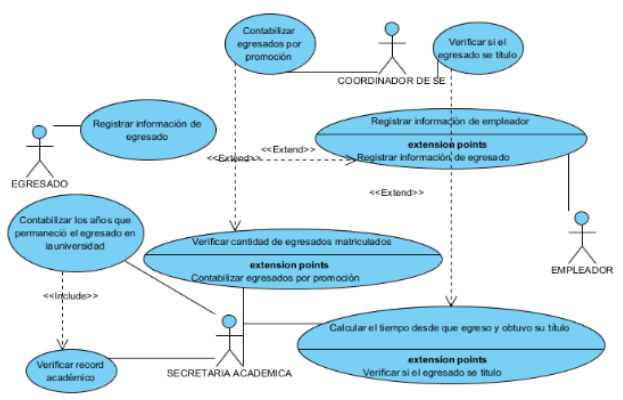

Tabla 6. Diagrama de CU Ingresar información de egresados

\begin{tabular}{|l|l|}
\hline Caso de uso & $\begin{array}{l}\text { Registrar información de } \\
\text { egresado }\end{array}$ \\
\hline Actor(es) & Egresado \\
\hline $\begin{array}{l}\text { Descripción: El caso de uso inicia cuando el } \\
\text { egresado ingresa al sistema a llenar su } \\
\text { información al sistema de seguimiento de } \\
\text { egresados. }\end{array}$ & $\begin{array}{l}\text { El Coordinador de } \\
\text { Seguimiento a egresados } \\
\text { invita al egresado a que } \\
\text { ingrese sus datos que el } \\
\text { sistema solicita. }\end{array}$ \\
\hline $\begin{array}{l}\text { Referencias } \\
\text { Precondiciones }\end{array}$ \\
\hline $\begin{array}{l}\text { Curso Básico } \\
\text { En caso contrario }\end{array}$ & $\begin{array}{l}\text { Si existe algún problema } \\
\text { en la carga de los datos el } \\
\text { sistema envía una } \\
\text { notificación }\end{array}$ \\
\hline $\begin{array}{l}\text { El egresado ingresa } \\
\text { datos que el } \\
\text { sistema le solicita. }\end{array}$ & $\begin{array}{l}\text { El sistema envía una } \\
\text { notificación de datos } \\
\text { correctamente. }\end{array}$ \\
\hline Acción del Actor \\
\hline \multicolumn{2}{|c|}{ Cursos Alternos } \\
\hline
\end{tabular}

Figura 8. Diagrama de CU del sistema Ingresar información

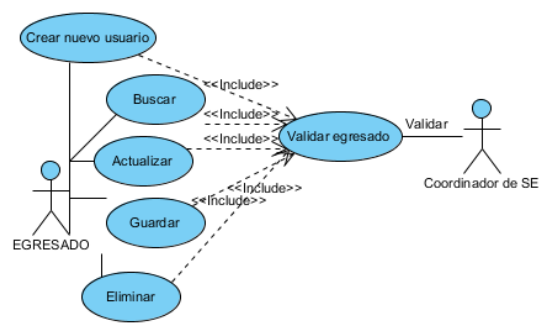

Tabla 7. Diagrama de CU Ingresar información de egresado

\begin{tabular}{|c|c|}
\hline Caso de uso & $\begin{array}{l}\text { Registrar información de } \\
\text { egresado }\end{array}$ \\
\hline $\operatorname{Actor}(e s)$ & Egresado \\
\hline \multicolumn{2}{|l|}{ Descripción: } \\
\hline \multicolumn{2}{|c|}{$\begin{array}{l}\text { El caso de uso inicia cuando el egresado ingresa } \\
\text { al sistema a llenar su información al sistema de } \\
\text { seguimiento de egresados. }\end{array}$} \\
\hline Referencias & - \\
\hline Precondiciones & $\begin{array}{l}\text { El Coordinador de } \\
\text { Seguimiento a } \\
\text { egresados invita al } \\
\text { egresado a que ingrese } \\
\text { sus datos que el sistema }\end{array}$ \\
\hline \multicolumn{2}{|c|}{ Curso Básico } \\
\hline $\begin{array}{l}\text { Acción del Actor } \\
\text { El egresado ingresa } \\
\text { los datos que el } \\
\text { sistema le solicita. }\end{array}$ & $\begin{array}{l}\text { Respuesta del Sistema } \\
\text { El sistema envía una } \\
\text { notificación de datos } \\
\text { procesados } \\
\text { correctamente. }\end{array}$ \\
\hline \multicolumn{2}{|c|}{ Cursos Alternos } \\
\hline En caso contrario & $\begin{array}{l}\text { Si existe algún } \\
\text { problema en la carga de } \\
\text { los datos el sistema } \\
\text { envía una notificación }\end{array}$ \\
\hline
\end{tabular}

Figura 9. Diagrama de CU del sistema -

Ingresar información de empleador 


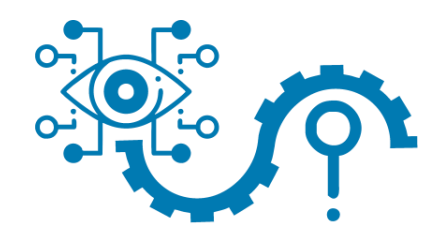

\section{GnasisWisdom}

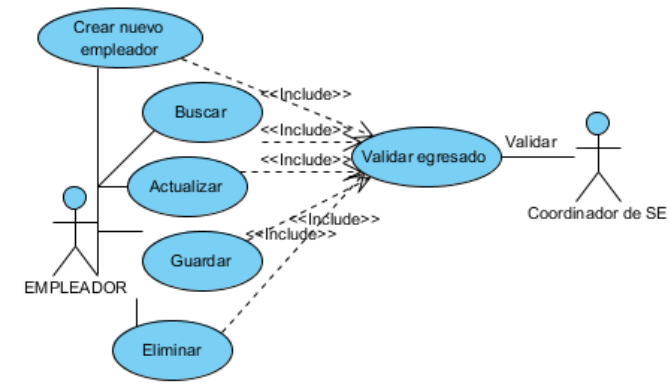

Tabla 8. Diagrama de CU Ingresar información de empleador

\begin{tabular}{|c|c|}
\hline Caso de uso & $\begin{array}{lll}\text { Registrar información } & \text { de } \\
\text { empleador } & & \\
\end{array}$ \\
\hline $\operatorname{Actor}(e s)$ & Empleador, Coordinador de SE \\
\hline \multicolumn{2}{|c|}{$\begin{array}{l}\text { Descripción: } \\
\text { El caso de uso inicia cuando el empleador ingresa } \\
\text { al sistema a llenar su información al sistema de } \\
\text { seguimiento de egresados. }\end{array}$} \\
\hline Referencias & 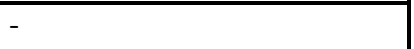 \\
\hline Precondiciones & $\begin{array}{l}\text { El Coordinador de } \\
\text { Seguimiento a egresados } \\
\text { invita al empleador que } \\
\text { ingrese sus datos que el } \\
\text { sistema solicita. }\end{array}$ \\
\hline \multicolumn{2}{|r|}{ Curso Básico } \\
\hline $\begin{array}{l}\text { Acción del Actor } \\
\text { El empleador } \\
\text { ingresa los datos } \\
\text { que el sistema le } \\
\text { solicita. }\end{array}$ & $\begin{array}{l}\text { Respuesta del Sistema } \\
\text { El sistema envía una } \\
\text { notificación de datos } \\
\text { procesados correctamente. }\end{array}$ \\
\hline \multicolumn{2}{|c|}{ Cursos Alternos } \\
\hline En caso contrario & $\begin{array}{l}\text { Si existe algún problema en la } \\
\text { carga de los datos el sistema } \\
\text { envía una notificación }\end{array}$ \\
\hline
\end{tabular}

\section{Discusión}

Con la implementación del Sistema de Seguimiento de Egresados se obtiene la información requerida de forma oportuna por parte de los egresados y empleadores,

\section{REVISTA DE INVESTIGACIÓN E INNOVACIÓN}

CIENTÍFICA Y TECNOLÓGICA

Volumen 1, Número 2, Mayo-Agosto 2021 Fecha de publicación: 10-05-2021 - Fecha de aceptación:20-04-2021

seguimiento a egresados, ya que en la Facultad de Ingeniería Electrónica Sistemas se realizaban el seguimiento a egresados de forma manual y no se contaba con los datos actualizados. $\mathrm{La}$ implementación del Sistema de Seguimiento de Egresados, ayudó a que ahora una forma de comunicación entre la institución y los egresados, posibilitando recabar información, lo cual es de suma importancia para el proceso de Licenciamiento $\mathrm{y}$ Acreditación.

Los beneficios de contar con un Sistema de Seguimiento de Egresados, se pueden clasificar como tangibles e intangibles, los primeros hacen mención a aquellos que pueden ser cuantificables y demostrados, $y$ el segundo, que a simple vista no se nota, tiene que ver con la percepción del usuario acerca del sistema. En la Tabla 10 se muestran los beneficios.

Tabla 10. Beneficios tangibles e intangibles

\begin{tabular}{|l|l|}
\hline Beneficios tangibles & \multicolumn{1}{|c|}{$\begin{array}{c}\text { Beneficios } \\
\text { intangibles }\end{array}$} \\
\hline $\begin{array}{l}\text { Ahorro de gastos por } \\
\text { concepto de materiales de } \\
\text { oficina y transporte, } \\
\text { reduciendo de esta manera } \\
\text { gastos operacionales. }\end{array}$ & $\begin{array}{l}\text { Mayor satisfacción } \\
\text { del usuario. }\end{array}$ \\
\hline $\begin{array}{l}\text { Mayor tiempo de } \\
\text { dedicación a labores } \\
\text { productivas por parte de } \\
\text { los usuarios, al no tener } \\
\text { que dedicar tiempo para } \\
\text { las entregas de los } \\
\text { informes. }\end{array}$ & \\
\hline
\end{tabular}
asimismo, se optimizó el proceso de 


\begin{tabular}{|l|l|}
\hline $\begin{array}{l}\text { Menores costos de } \\
\text { operación. }\end{array}$ & $\begin{array}{l}\text { Garantía } \\
\text { objetividad en la } \\
\text { automatización del } \\
\text { sistema. }\end{array}$ \\
\hline $\begin{array}{l}\text { Generación de reportes } \\
\text { automáticos. }\end{array}$ & $\begin{array}{l}\text { Disponibilidad } \\
\text { inmediata de la } \\
\text { información. }\end{array}$ \\
\hline $\begin{array}{l}\text { Garantía } \\
\text { confidencialidad } \\
\text { seguridad. }\end{array}$ & $\begin{array}{l}\text { El uso correcto del } \\
\text { sistema a través de } \\
\text { los usuarios. }\end{array}$ \\
\hline $\begin{array}{l}\text { Elaboración de procesos } \\
\text { en menor tiempo. }\end{array}$ & $\begin{array}{l}\text { Sistema } \\
\text { automatizado de } \\
\text { procesos }\end{array}$ \\
\hline $\begin{array}{l}\text { Disminución de los } \\
\text { tiempos de respuesta } \\
\text { relacionados con las } \\
\text { consultas y envíos de } \\
\text { información. }\end{array}$ & $\begin{array}{l}\text { Mayor } \\
\text { conocimiento de la } \\
\text { situación laboral de } \\
\text { los egresados. }\end{array}$ \\
\hline
\end{tabular}

\section{Conclusiones}

1. El sistema de seguimiento a egresados es una herramienta fundamental para evaluar la pertinencia de la formación profesional del egresado y su satisfacción.

2. El sistema de seguimiento a egresados permite medir el nivel de desempeño en el centro de trabajo.

3. A través de las historias de usuario, herramienta brindada por la metodología XP, se facilitó la recolección de la información necesaria para definir los requisitos del sistema, mediante el contacto y comunicación directa con el cliente, permitiendo además retroalimentación en la misma.

4. El adecuado diseño de la base de datos resultó fundamental en el desarrollo del software, ya que el mismo sirvió de base para constituir la arquitectura del

\section{REVISTA DE INVESTIGACIÓN E INNOVACIÓN}

CIENTÍFICA Y TECNOLÓGICA

Volumen 1, Número 2, Mayo-Agosto 2021 cha de publicación: 10-05-2021 - Fecha de aceptación:20-04-2021 sistema, evitando incrementar costos y gastos innecesarios. Una aplicación que además de abarcar y controlar todo lo necesitado por el usuario y/o cliente, representa un sistema bastante completo que además otorga información real y exacta para ser usada como sustento en la toma de decisiones.

\section{Referencias:}

Beck, K. (2004). Extreme Programming Explained. Embrace Change. (Segunda Edición). Editorial Addison Wesley.

Calero, M. (2003). Una explicación de la Programación Extrema (XP).

Elmasri, R. y Navathe, S. (2000). Sistemas de Bases de Datos. Conceptos Fundamentales. (Segunda Edición). México, D.F.: Editorial Addison - Wesley Iberoamericana.

Hernández, R.; Fernández, C. y Baptista, P. (2006). Metodología de la Investigación. México, D.F.: Editorial McGrawHill.

INEI (2014). Encuesta Nacional a egresados universitarios.

Kendall, K. y Kendall, J. (2005). Análisis y Diseño de Sistemas. (Sexta Edición). México, D.F.: Editorial Pearson.

Leiva, I. L., y Moreno, C. F. (2015). Implementación de un Sistema Virtual de Seguimiento de Egresados en la Facultad de Ingeniería Industrial, Sistemas e Informática de la Universidad Nacional José Faustino Sánchez Carrión. Tesis. UNIVERSIDAD 


\section{GnosisWisdam}

NACIONAL "JOSÉ FAUSTINO

SÁNCHEZ CARRIÓN."

Porozo, E. L. (2014). Diseño e

Implementación de un Sistema Web de

Seguimiento a Graduados. Tesis.

UNIVERSIDAD DE GUAYAQUI.

Pressman, R. (2002). Ingeniería del software, un enfoque práctico. (Quinta Edición). Madrid, España: Editorial McGrawHill.

SINEACE. (2016). Modelo de Acreditación para programas de Estudios de Educación Superior Universitaria, Disponible en https://www.sineace.gob.pe/acreditacion/un iversidades/

Sommerville, I. (2005). Ingeniería del Software. (Séptima Edición). Madrid, España: Editorial Pearson.

Universidad San Martín de Porres (2016).

Sistema de medición de la empleabilidad.

\section{Correspondencia}

Mg. Freddy Toribio Huayta Meza

fhuaytam@hotmail.com 